knowledge we must set the more systematic training to which students are subjected before they are expected to take an independent line; secondly, the new methods of treatment and new points of view which now succeed one another more rapidly than at any previous time; and, thirdly, the very greatly increased facilities for research on the spot in foreign countries. When it is remembered how many of our most prominent men started their careers as travellers, the importance of the third of the above considerations will be valued as it ought to be. Those who are best able to appreciate the position of anatomical and physiological botany would probably be the first to agree that the opportunities for research in these branches, either in foreign lands or at home, are, at the present moment, better than they have been at any former period in the history of the science. If the botanical students of the present day content themselves with devoting their time and energy to working out small and uninteresting details, it is their own poverty of imagination and want of enterprise that are to blame.

F. O. B.

\section{MOLECULAR DYNAMICS}

I HAVE placed the three titles above this article not because I intend to deal with more than the last, but because they all deal with the same matter, and show how much the author's attention was directed to the subject during his three months' sojourn in America. The audience at the Baltimore lectures consisted chiefly of American professors, and a few English men of science attended a larger or smaller number of the lectures.

Speculation was rife as to the probable character of the lectures, and there was a general feeling that vortex motion would be largely dealt with. This, however, was not so. The course of twenty lectures was confined to the wave theory of light, largely dealing with the difficulties of that theory. The published lectures are not printed, but "jelligraphed," as Sir William Thomson would say. The number of copies is extremely limited, and are of unique interest, being reproduced from the short-hand notes taken at the lectures. Every one who knows how suggestive Sir William's lectures are and how fertile his mind is in bringing illustrative digressions to bear on the topic in hand, will expect these verbatim notes to be a rare treasure. Nor will he be disappointed. Mr. Hathaway, the reporter, has the unusual combination of being an expert stenographist, a skilful mathematician, and a clear and distinct caligraphist. His notes contain numerous errors, such as are unavoidable in such an undertaking, but, viewed as a whole, his work is almost a marvel.

The lectures treated of three branches of the subject: (I) the propagation of a disturbance through an elastic medium ; $(2)$ the character of molecular vibration; and (3) the influence of molecules on the propagation of waves. Each lecture generally dealt with two of these branches, and between the two parts of the lecture Sir William went among his andience and had some conversation with them. It was ever his object to discard the professorial attitude and give his lectures the aspect of conferences. Discussion did not end in the lecture-room, and the three weeks at Baltimore were like one long conference guided by the master mind. It is not surprising that at the end of that time there was a genuine feeling of sadness at parting on the part of teacher and taught alike.

The part of the lectures dealing with the propagation of an elastic disturbance could not be expected to contain

I "On Molecules," the Presidential Address to Section A of the British Association, August, 188 4. by Sir William Thomson.

"The Wave Theory of Light," a Lecture delivered by Sir William Thomson at Philadelphia on Sept. 29 , 1884 , published in NATURE, vol. xxxi p. gI.

Hopkins University Octuber, Dynami much novelty, but it was treated in so novel a manner and from so purely a physical point of view, that it could not but be instructive. Many of the old supporters of the theory dealt with it purely from a mathematical point of view. They treated the problem as a mathematical exercise, and did not hesitate to make unwarranted assumptions to produce pretty formulas or simple solutions. Even such men as Weber (in his "Theory of Magnetism ") and Green (in his "Wave Theory") have been guilty of this practice. Sir William Thomson never made any but physical assumptions, and these were made for reasons given. Rather than make a meaningless mathematical assumption he would prefer to burden his formulas with undetermined quantities, and even, if unable to reach the final solution, would rejoice in the richness of the formulas, which showed a potentiality of overcoming many difficulties. He does not always commend Rankine's mathematics, but he says this for him at p. I85: "Rankine did a great deal to cure the mathematical disease of asphasia from which we suffered so long. Faraday did most. The old mathematicians used neither diagrams to help people to understand their work, nor words to express their ideas. It was formulas, and formulas alone. Faraday was a great reformer in that respect with his language of 'lines of force,' \&c. Rankine was splendid in his vigour and in the grandeur of his Greek derivations." This refers to Rankine's nomenclature of different kinds of moduluses and their reciprocals-e.g. plagiotatic, thlipsinomic, \&c.

The first lecture is a summary of what is to come, and is partly historical. The difficulties in the way of accepting the wave theory of light are clearly pointed out. These are four in number.

First Difficulty: Dispersion.-The difficulty is to explain how velocity of propagation depends on period of vibration. Two explanations have been offered, that of Cauchy and that of Helmholtz. He does not delay much with Cauchy, who ascribed it to heterogeneousness. He prefers Helmholtz, who ascribes it to a compound structure of material molecules, which gives them a natural period of vibration. The one explanation has relation to wave-length, the other to period of vibration. The latter, he thinks, falls in better with results of spectrum analysis, \&c. A great portion of the lectures is devoted to expanding the notion of Helmholtz. The space occupied by a molecule must be filled with a substance differing from the ether either in rigidity or in density, or both. Lord Rayleigh has taken in hand this question in his researches on blue sky, and it seems that a variation of density is the principal or only effective cause. With respect to the new (Helmholtz-Thomson) spring and shell molecule, he says, "It seems to me that there must be something in this, that this, as a symbol, is certainly not an hypothesis, but a certainty."

Second Difficulty: the Ether.-He makes short work of the difficulty of reconciling almost perfect rigidity with almost perfect mobility. It is merely a matter of time. You can make a tuning-fork of Burgundy pitch when the period is a small fraction of a second, but a bullet will pass clean through several inches of it in six months. The ether may be highly elastic for vibrations executed in the 100 or 1600 million millionth of a second, but highly mobile to bodies going through it at the rate of twenty miles a second.

Third Difficulty: Refraction and Reflection.-Theoretical equations agree qualitatively with facts, but there are serious discordances when we come to quantitative measurements. Especitlly is this the case in the completeness of extinction of the ray polarised by reflection.

Fourth Difficulty: Double Refraction.-It is found that when the medium is displaced during wave-propagation in a double refracting crystal, the return force must depend on the direction of vibration, not on the plane of distortion, as all elastic theories indicate. Rankine and 
Rayleigh independently invented unequally loaded molecules which overcome the difficulty, but give a wave surface different from Huyghens', and Stokes has proved experimentally that Huyghens' construction is very accurate. Hence this way of escape is denied to us.

In treating of the propagation of waves in an isotropic (and later in an aeolotropic) medium, methods of Thonson and Tait's Natural Philosophy, and his own article, "Elasticity," in the "Encyclopædia Britannica," are used; $a b c$ are distortions about $O x, O y$, and $O z$, ef $y$ are dilatations along $O x, O y, O z$. The equation of the energy $E$ is a quadratic in $a b c e f g$, containing twenty-one coefficients, some of which are annulled by isotropy.

If

$$
\begin{aligned}
& P=\frac{d E}{d e}, Q=\frac{d E}{d f}, R=\frac{d g}{d E} ; \\
& S=\frac{d E}{d a}, T=\frac{d E}{d b}, U=\frac{d E}{d c},
\end{aligned}
$$

and if $\rho$ be the density, and $\xi$ a displacement aiong $O x$, we obtain the equation

$$
\rho \frac{d^{2} \xi}{d t^{2}}=\frac{d P}{d x}+\frac{d U}{d y}+\frac{d T}{d z} .
$$

Moreover, if $n$ be the rigidity modulus, $k$ the bulk modulus, $\delta$ the cubic dilatation, and $m=\vec{k}+\frac{1}{3} n$, we have

$$
\begin{gathered}
P=(m-n) \delta, \\
T=n\left(\frac{d \xi}{d z}+\frac{d \zeta}{d x}\right),
\end{gathered}
$$

with similar expressions for $Q, R, S, U$.

Thence he shows that the cquation

$$
\rho \frac{d^{2} \xi}{d t^{2}}=m \frac{d \delta}{d x}+n \nabla^{2} \xi
$$

(with the condition $\frac{d u}{d x}+\frac{d v}{d y}+\frac{d w}{d z}=0$ in an incom-

pressible substance), contains every possible solution, and he proceeds to discuss special cases of the general solution which may be true of waves propagated by molecules through the ether. Here his desire for physical conceptions appears, and his hatred of mathematical asphasia. $\mathrm{He}$ considers the case of a ball moving to and fro, of a ball twisting about an axis, of a globe becoming alternately prolate and oblate, of a rod twisted in opposite directions at the two ends, and of the Thomson-Helmholtz molecule which is a heavy mass connected by massless springs with a massless inclosing shell, or there may be several shells inclosing each other, connected by springs with a dense mass in the centre (far more dense than the ether).

Here he discusses the manner in which a molecule may be supposed to give off its vibrations to the ether. Does it gradually increase in intensily and gradually die out, or how does it act? Here is what he says on this muchneglected point at p. $94:-$

"The kind of thing that the luminous vibrator consists in seems to whe to be a sudden initiation of a set of vibrations and a sequence of vibrations from that initiation which will naturally become of smaller and smaller amplitude. .. . Why a sudden start? Bccause I believe that the light of the natural flame or of the arc light or of any other known source of light must be the result of sudden shocks from a number of vibrators. Take the light obtained by striking two quartz pebbles together. You have all scen that. There is one of the very simplest sources of light. . . . What sort of a thing can the light be that proceeds from striking two quartz pebbles together? Under what circumstances can we conccive a. group of waves of light to begin gradually and to end gradually? You know what takes place in the excitation of a fiddle-string or a tuning-fork by a bow. The vibrations gradually get up from zero to a maximum, and then, when you take the bow off, gradually subside. I cannot sec anything like that in the source of light. On the contrary, it secms to me to be all shocks-a sudden beginning and gradual subsidence."

The light coming from a single shock is, of course, polarised always in the same direction. Sellmeier's deductions from Fizeau's experiment shows that there is no serious fading in 50,000 vibrations. Helmholtz introduces viscous terms which absorb the energy and might prevent the possibility of 50,000 vibrations from one shock. That is a retrograde step. Absorption can be explained without viscous terms.

Such speculations, when coming from one of less grasp of physical facts, would attract but little attention. But here all kinds of useful suggestions are continually thrown out for experiment and for hypotheses. He is striving to get at the physical meaning of radiation, absorption, anomalous dispersion, fluorescence, and phosphorescence, and here is what he says on some of these points at p. $90:-$

"But there are cases in which we have that tremendous jangling, and that is in the fluorescence of such a thing as uranium glass, which lasts for several seconds after the exciting light is taken away, and then again in phosphorescence that lasts for hours and days. There have been exceedingly interesting beginnings in the way of experiments already made, but I think no one has found whether initial refraction is exactly the same as permanent refraction. For this purpose we might use Becquerel's phosphoroscope, or we might take such an appliance as Prof. Michaelson has been using for light, and get something enormously more searching than Becquerel's phosphoroscope, and try whether, in the first hundredth of a second, there is any indication of a different wave-velocity from that which you would have when white light passes continuously in the usual manner of refraction. If in the methods employed for ascertaining the velocity of light in a transparent body . . . we apply a test for an instantaneous refraction, I have no doubt we shall get negative results, but yet properties of ultimate importance. We might take bodies in which, like uranium glass, the phosphorescence lasts only a $f \in w$ seconds; and then, again,
bodies in which phosphorescence lasts for minutes and hours. With some of these we should have anomalous dispersion, gradually fading away after a time. I should think that by experimenting, and so on, we should find some very interesting results of this kind."

In his mathematics he suppresses the condensational wave, and, in doing so, makes reference to the electromagnetic theory of light, which, he thinks, has added nothing to our physical conceptions of the ether. In treating, further on, of reflcction and refraction, he speaks a great deal of the prcssural wave, which many authors have called a condensational wave. I find that in some points my notes are fuller than the reporter's. I cannot find there the following characteristic passage about the pressural wave:-- "People have tried to muddle this. The prossural wave has been the difficulty. Cauchy starved the animal, M'Cullagh and Neumann didn't know of its existence, Haughton put it in an Irish car and it wouldn't go, Green and Rayleigh treated it according to its merits."

With regard to the possibility of a condensational wave, and to the electro-magnetic theory of light, we find, on pp. $40.41:-$

"We ignore this condensational wave in the theory of light. We are sure that its energy, at all events, if it is not null, is very small in comparison with the luminiferous vibrations we are dealing with. But to say that it is absolutely null would be an assumption we have no right to make. When we look through the little universe that we know, and think of the transmission of electrical force and of the transmission of magnetic force, and of the transmission of light, we have no right to assume that there is not something else that our philosophy does 
not dream of. We have no right to assume that there may not be condensational vibration in the luminiferous ether. We only do know that any vibrations of this kind which are excited by the reflection and refraction of light are certainly of very small energy compared with the energy of the light from which they proceed. The fact of the case as regards reflection and refraction is this: that, unless the luminiferous ether is absolutely incompressible, the reflection and refraction of light must generally give rise to waves of condensation. Waves of distortion may exist without waves of condensation, but waves of distortion cannot be reflected at the boundary surface between two mediums without exciting in each medium a wave of condensation. When we come to the subject of reflection and refraction we shall see how to deal with these condensational waves, and find how easy it is to get quit of them by supposing the medium incompressible. But it is always to be kept in mind to be examined into: Are there or are there not very small amounts of condensational waves generated in reflection and refraction; and may, after all, the electric force not depend on the waves of condensation? Suppose that we have at any place in air, or in luminiferous ether, a body that, through some action we need not describe, but which is conceivable, is alternately positively and negatively electrified : may it not be that this will be the cause of condensational waves?" It is then supposed that two spherical conductors are connected to the terminals of an alternating dynamo machine, and the quotation proceeds:-

"It is perfectly certain, if we turn the machine slowly, that in the neighbourhood of the conductors we will have alternately positively and negatively electrified elements with reversals perhaps two or three hundred per second of time, without a gradual transition from negative through zero to positive, and the same thing all through space; and we can tell exactly what is the potential at each point. Now, does any one believe that, if that revolution was made fast enough, the electrostatic law would follow? Every one believes that, if that process be conducted fast enough several million times, or millions of million times per second, we should be far from fulfilling the electrostatic law in the electrification of the air in the neighbourhood. It is absolutely certain that such an action as that going on would give rise to electrical waves. Now it does seem probable to me that electrical waves are condensational waves in luminiferous ether, and probably it would be that the propagation of these waves would be enormously faster than the propagation of ordinary light waves. I am quite conscions, when speaking of this, of what has been done in the so-called electro-magnetic theory of light. I know the propagation of electric impulse along an insulated wire surrounded by gutta-percha, which I worked out myself about the year I854, and in which I found a velocity comparable with the velocity of light. . . That is a very different case from this, and I have waited in vain to see how we can get any justification of the way of putting it in the socalled electro-magnetic theory of light."

In those parts of the lectures which deal with wave propagation in an isotropic medium, by far the most interesting parts are those which treat of the conditions at bounding surfaces, whether these surfaces be reflecting and refracting surfaces or surfaces of radiating molecules, or surfaces of absorbing molecules. Lord Ravleigh's investigations and his own on the likelihood of the density or the rigidity of the substance composing a molecule differing from that of the ether are also full of interest

Much of this part of the subject has been thoroughly worked out before, but here the treatment is so original, the language is so suggestive, and I need hardly say that the whole course of lectures is so pregnant with useful ideas, that every one who reads this part will be well repaid.
Having now roughly indicated the novel points and the general mode of treatment of the problem in molar dynamics, I propose in the next notice to give some account of the problem in molecular dynamics, which occupied half of the time. GEORGE FORBES

( $T o$ be continued.)

\section{THE LONG DURATIONS OF METEORIC RADIANT POINTS}

$\mathrm{I} \mathrm{T}$ is unfortunate that the observation of shooting stars is associated with difficulties of no common order. The very large number of distinct showers visible at the same epoch, their extremely attenuated character, and the many impediments to accurate determinations of the flights of the individual shooting stars proceeding from them, exercise an unfavourable influence on the work and deter many observers from grappling with a subject which is admittedly beset with such perplexing details. Apart from this, there exists the great necessity for observations to be sustained during the whole night, and this is rarely practicable either by amateur or professional astronomers, who generally have other important work in hand. In fact, meteoric astronomy requires the almost exclusive attention of the observer, and must be closely pursued for a long period of time if anything like comprehensive results are to be obtained. The voids occasioned either by moonlight or cloudy weather in a short series of observations are only to be filled up by prolonged watches extended over many consecutive years.

The long visible duration of a large number of radiant points of shooting stars is, it must be confessed, a fact which defies satisfactory explanation. The ingenious theory which had attributed to meteor streams an identity with cometary orbits, required that the visibility of such streams should be of very brief character, though in the case of an abnormally wide system or of a shower directed from a point near the earth's apex the duration might be longer than usual, but the radiant point could not maintain a perfectly fixed position amongst the stars. This general view of the subject is, however, not accordant with the results of recent observations, for while there are undoubtedly some cometary showers which display all the peculiarities taught by theory, there are many other streams which continue visible for several months and retain a stationary position in the firmament. It is evident therefore that these streams are presented to us under totally different circumstances as regards orbit to the true planetary showers, and are amenable to conditions and laws which form a problem the solution of which is arrested by no ordinary difficulties.

The multiplicity of streams would naturally originate a false appearance of long duration in certain radiant points, but observations of very precise character would soon show that the point of radiation, as successively determined, differed considerably, being not, in fact, confined absolutely to the same point in the sky. But it is now proved that there are no differences, other than those introduced by small unavoidable errors of observation, in the centres from which shooting stars continue to fall during several months. Indeed, it seems a probable inference from the observations that some showers exist all the year round, though not visible during the epoch when they are very near to the sun.

That such long enduring radiants of meteors can have a community of origin and belong to physically associated streams in the same degrce as the true cometary meteor showers is very difficult to understand. But the fixity of the radiant over so long an interval would yet seem to indicate some bond of close affinity existing between them. At any rate we have no reason to suppose that a large number of showers, distinct in themsclves, can occur consecutively from the same points of the sky owing to a common peculiarity of grouping. 